\title{
Do high risk patients alter their lifestyle to reduce risk of colorectal cancer?
}

\author{
Gregory P Tarr ${ }^{1}$, Andrew Crowley ${ }^{1}$, Rhys John ${ }^{1}$, Jonathan B Kok ${ }^{1}$, Ho-Nam L Lee ${ }^{1}$, Hasif Mustafa ${ }^{1}$, Kia M Sii ${ }^{1}$ \\ Rebecca Smith', Sung-Eun Q Son ${ }^{1}$, Lauren J Weaver ${ }^{1}$, Claire Cameron², John D Dockerty ${ }^{2}$, Michael Schultz ${ }^{1,3^{*}}$ \\ and lain A Murray ${ }^{3}$
}

\begin{abstract}
Background: Colorectal cancer (CRC) may be reduced by healthy lifestyle behaviours. We determined the extent of self-reported lifestyle changes in people at increased risk of CRC, and the association of these reports with anxiety, risk and knowledge-based variables.

Methods: We randomly selected 250 participants who had undergone surveillance colonoscopy for family history of CRC. A telephone interview was conducted, recording demographics and family history. Self-reported lifestyle change due to thoughts about CRC across a range of dietary and lifestyle variables was assessed on a four-point scale. Participants' perceptions of the following were recorded: risk factor knowledge, personal risk, and worry due to family history. General anxiety was assessed using the GAD-7 scale. Ordinal logistic regression was used to calculate adjusted results.

Results: There were 148 participants (69\% response). 79.7\% reported at least one healthy change. Change in diet and physical activity were most frequently reported (fiber, 63\%; fruit and vegetables, 54\%; red meat, 47\%; physical activity, 45\%), with consumption of tobacco, alcohol, and body weight less likely (tobacco, 25\%; alcohol, 26\%; weight 31\%). People were more likely to report healthy change with lower levels of generalized anxiety, higher worry due to family history, or greater perceived knowledge of CRC risk factors. Risk perception and risk due to family history were not associated with healthy changes.
\end{abstract}

Conclusions: Self-reported lifestyle changes due to thoughts about CRC were common. Lower general anxiety levels, worries due to family history, and perceived knowledge of risk factors may stimulate healthy changes.

Keywords: Colorectal cancer, Surveillance, Healthy lifestyle

\section{Background}

Colorectal cancer (CRC) is a major cause of morbidity and mortality, and this burden may be reduced by the adoption of a range of healthy behaviours, including screening/ surveillance, and a choice of lifestyle factors [1].

Changing patterns of screening behaviour and healthy lifestyle factors have been linked to decreased incidence of CRC. Since 1975, CRC incidence has fallen by around $30 \%$ in the United States. This reduction appears to be equally attributable to increased screening/surveillance, and to adopting healthy lifestyle behaviours [2].

\footnotetext{
*Correspondence: michael.schultz@otago.ac.nz

'Dunedin School of Medicine, University of Otago, PO Box 913, Dunedin, New Zealand

${ }^{3}$ Gastroenterology Unit, Dunedin Public Hospital, Dunedin, NZ

Full list of author information is available at the end of the article
}

Cancer risk factors are often reported in popular media, and it appears that public knowledge of CRC risk factors $[3,4]$ parallels those for cardiovascular disease $[5,6]$. In a large Dutch cohort, increasing prevalence of healthy lifestyle behaviours was associated with a reduction in incidence of CRC [7]. At the population level, moderate variation in levels of exercise, tobacco smoking, fruit/vegetables/fiber and alcohol intake appeared to explain around one quarter of the incidence of CRC [7].

Current strategies for modifying healthy lifestyles have had some success in improving risk profiles [8]. People undergoing CRC screening appear to spontaneously adopt healthy lifestyle behaviours for at least a short time around the time of screening [9], suggesting that concerns about CRC may provide a stimulus for positive

\section{Biomed Central}

(C) 2014 Tarr et al.; licensee BioMed Central Ltd. This is an open access article distributed under the terms of the Creative Commons Attribution License (http://creativecommons.org/licenses/by/2.0), which permits unrestricted use, distribution, and reproduction in any medium, provided the original work is properly cited. 
choices, and people are adherent to screening recommendations are also more likely to adhere to other healthy behaviours [10]. A substantial body of research has linked various factors with the uptake of screening. Risk perception [11] and knowledge [12] both influence the choice to be screened, and multiple studies have linked general anxiety symptoms to increased screening uptake [13-16], although this has been disputed [17-19]. Cancer-specific worry may be a better predictor of screening [20].

While screening and surveillance are important factors in reducing the burden of $\mathrm{CRC}$, lifestyle based harm reduction strategies will likely also play an important part [21]. In light of these findings, it is important to know what promotes people to adopt healthy lifestyles, especially in high risk groups such as familial CRC. However, literature on this is sparse. Qualitative studies highlight the current mismatch in public understanding of CRC, with patients undergoing CRC screening perceiving adenomas as a minor problem [22], having lack of understanding of CRC risk factors [23] and some being skeptical about the need for lifestyle change [22,23]. On a more positive note, some patients were amenable to changing their lifestyles in order to decrease risk of CRC $[22,23]$.

The aims of our study were to describe the extent of self-reported changes in healthy lifestyle behaviours in a patient group at increased risk of CRC, and the effect risk perception, anxiety and family history of CRC had on the adoption of these lifestyles.

\section{Methods}

\section{Study population}

Patients who underwent colonoscopy surveillance between 1996 and 2012 at Dunedin Hospital, New Zealand for a family history of CRC were eligible for inclusion. Participants were excluded if they were aged over 75 years at recruitment (the age at which surveillance colonoscopy is discontinued, in keeping with national guidelines), had a personal history of CRC, symptoms of CRC or inflammatory bowel disease. Participants were randomly selected from a clinical endoscopy database of 1,086 patients.

\section{Study questionnaire}

Potential participants were provided with an information sheet and visual aid by mail, then telephoned to obtain verbal consent. The survey was administered using a labeled visual scale to assist in risk estimation. The telephone interview was standardized across ten interviewers. The methods are reported previously elsewhere [24]. Briefly, demographic and clinical details were collected during the interview, and additional clinical details were obtained from hospital records.

Demographic information is shown in Table 1. Participants reported family history of cancer for first- and
Table 1 Demographic and clinical characteristics of the study population

\begin{tabular}{lc}
\hline & $\mathbf{N}=\mathbf{1 4 8}$ \\
\hline Age, years & $57.9(56.5$ to 59.3$)$ \\
NZ European & $56(37.8 \%)$ \\
NZDep2006 + & $142(96.0 \%)$ \\
Alcohol, median (IQR) $\neq$ & $5.3(4.9$ to 5.7$)$ \\
No alcohol use & $3(1-7)$ \\
Tobacco use & $36(24.3 \%)$ \\
$\quad$ Never & \\
Ex & $68(46.3 \%)$ \\
Current & $66(44.9 \%)$ \\
\end{tabular}

Marital status

Married/de facto

109 (73.6\%)

Single/widowed

$22(14.9 \%)$

Divorced/separated

$17(11.5 \%)$

Education completed

$\begin{array}{lc}\text { Primary } & 6(4.1 \%) \\ \text { Secondary } & 75(50.7 \%) \\ \text { Tertiary } & 66(44.6 \%) \\ \text { Personal history of non-CRC cancer } \S & 23(15.5 \%)\end{array}$

Colonoscopies, median (IQR)

$1(1-3)$, range $1-7$

Polyps removed, median (IQR)

First degree relatives, median (IQR)

$1(0-2)$, range $0-120$

$1(1-2)$

Age of first degree relative at diagnosis

Second degree relatives, median (IQR)

49.5 (47.5 to 51.6$)$

$1(0-2)$

Total relatives with any cancer,

$3(2-4)$

median (IQR) ††

NZGG category 1

( 12\% lifetime risk)

$15(10.1 \%)$

NZGG category 2

$66(44.6 \%)$

( 18 to $36 \%$ lifetime risk)

$57(38.5 \%)$

NZGG category 3

(up to $50 \%$ or greater lifetime risk)

Second degree relatives only

$10(6.8 \%)$

(risk approximately 5.9\%)

Results are mean (95\% confidence interval or number (percentage), unless stated otherwise.

† New Zealand Deprivation Index 2006.

₹ Units of alcohol consumed in one average week.

§ Participants with a history of CRC were excluded.

Number of relatives with CRC.

t† Number of relatives (first- or second-degree) with any cancer.

$\mathrm{CRC}=$ colorectal cancer; $\mathrm{IQR}=$ interquartile range; $\mathrm{NZGG}=$ New Zealand

Guidelines Group.

second-degree relatives, including type of cancer and age at diagnosis, confirmed by review of hospital records. The number and year of surveillance colonoscopies were recorded from hospital records including colonoscopy outcomes. NZDep2006 is a measure of socioeconomic 
deprivation derived from census indices, based on the street address of each participant [25].

Each participant was asked for their perception to what degree CRC is genetic, how knowledgeable they were about CRC lifestyle risk factors, and to what degree they had altered the following lifestyle risk factors (reducing weight, red/processed meat, alcohol and smoking; increasing dietary fiber, fruit/veges, and physical activity) because of concerns about CRC. Participants were prompted with regard to each lifestyle factor (i.e. "how much have you reduced tobacco/cigarette consumption because of thoughts about CRC"). We used a four point, ordinal scale (Not at all, A little, Somewhat, Very much so). The questions regarding behavioural change enquired about subjective, self-perceived change since the initiation of surveillance. There was no formal validation of patient responses to measured behaviours. For patients who did not smoke/ consume alcohol, their change in behaviour was labelled as "Not applicable" We asked participants to estimate their personal lifetime risk of CRC without colonoscopy surveillance on a $0-100 \%$ scale. The Generalized Anxiety Disorder 7 scale (GAD-7) was administered as per authors recommendations [26].

The Lower South Regional Ethics Committee approved the study.

\section{Study measures}

Risk of CRC was estimated by New Zealand Guidelines Group (NZGG) recommendations [27]. These guidelines classify risk by the presence of family history and features of genetic CRC syndromes. Briefly, NZGG categorizes patients into four risk categories on the basis of the number of first degree relatives with histories of CRC and their age at first diagnosis [27]. NZGG category was determined by a team of three investigators (GPT, AC, JR) and from clinical documentation, and agreement between the two was good (Kappa 0.76).

\section{Statistical analysis}

Statistical analysis was performed using Stata 10.0 (StataCorp) and StatView version 5.01 (SAS Institute). Results are shown as mean ( $95 \%$ confidence interval) or number (percentage) unless otherwise stated. Continuous variables were compared between categories using ANOVA, and discrete variables were compared using the Chi-square test. To assess the relationship between anxiety, worry due to family history, and perceived knowledge and behavioural change, ordinal logistic regression including adjustments for age, sex and socioeconomic status was performed. A P value of $<0.05$ was considered statistically significant. This was an exploratory study, and there was no previous data to base a sample size calculation on. Pearsons correlation was used to measure the association between different the number of healthy lifestyles adopted, and anxiety, worry and knowledgeability.

The primary outcome was the proportion of participants reporting changes in each healthy behaviour. Secondary outcomes included whether risk perception, generalized anxiety, worry due to family history and risk due to family history were associated with changes in a composite of all recorded healthy behaviours. To adjust these analyses for confounding factors, ordinal logistic regression was used. Odds ratios compared either a onepoint increase, or SD increase of exposure variable with the number of healthy lifestyle behaviours changed "Somewhat" or "Very much so" compared to those changed "Not at all" or "A little".

\section{Results}

\section{Recruitment and demographics}

Full details of the study population including demographics are reported elsewhere [24]. Of 250 people initially sampled, 34 were ineligible, 47 could not be contacted and 21 declined consent. The exclusions were as follows: previous CRC $n=11$, inflammatory bowel disease $\mathrm{n}=4$, not in surveillance program $=1$, duplicate $\mathrm{n}=1$, deceased $\mathrm{n}=4$, aged over 75 years $\mathrm{n}=13$. The remaining 148 (69\%) participated. Those excluded were younger than respondents ( 55.3 years old [95\% CI 53.0 to 57.5 ] vs. 57.9 [95\% CI 56.5 to 59.3 ]; $\mathrm{p}<0.05$ ), but similar by sex $(42.4 \%$ vs. $37.8 \%$ male; $\mathrm{P}=0.63)$. The clinical and demographic details including NZGG-based risk groups of the included population are given in Table 1. The majority were middle aged (range 35 to 74 years), female, married or in a de-facto relationship, had undergoing a single colonoscopy and were at least three times the population risk of developing colorectal cancer in their lifetimes.

\section{Self-reported knowledge of colorectal cancer risk factors and behavioural change}

The majority of participants $(77.7 \%)$ believed that CRC risk was genetic and relatively uninfluenced by lifestyle behaviours (Table 2). Two-thirds of patients worried "Sometimes", "Often" or "Always" about their risk based on their family history. The majority felt they were "Somewhat" or "Very much so" aware of lifestyle risk factors for CRC.

Most $(79.7 \%, \mathrm{n}=118)$ reported altering at least one lifestyle variable to reduce their CRC risk (Figure 1). For most, this meant dietary change. Thirty participants (20.2\%) reported no changes. The median number of healthy behaviours adopted was two. A small number of participants $(4.1 \%, \mathrm{n}=6)$ reported adopting all healthy behaviours at least somewhat. Relatively few reduced their weight, alcohol intake or tobacco use due to concern over CRC risk. 
Table 2 GAD-7 score, anxiety about surveillance, and worry about family history of colorectal cancer

\begin{tabular}{|c|c|}
\hline & $N=148$ \\
\hline \multicolumn{2}{|l|}{$\begin{array}{l}\text { To what extent is CRC genetic and will } \\
\text { happen regardless of how we live? }\end{array}$} \\
\hline Not at all & $4(2.7 \%)$ \\
\hline A little & $29(19.6 \%)$ \\
\hline Somewhat & $62(41.9 \%)$ \\
\hline Very much so & $53(35.8 \%)$ \\
\hline \multicolumn{2}{|l|}{$\begin{array}{l}\text { Do you worry about developing CRC } \\
\text { based on your family history? }\end{array}$} \\
\hline Not at all & $18(12.2 \%)$ \\
\hline A little & $36(24.3 \%)$ \\
\hline Sometimes & $62(41.9 \%)$ \\
\hline Often & $25(16.9 \%)$ \\
\hline Always & $7(4.7 \%)$ \\
\hline \multicolumn{2}{|l|}{ I am aware of lifestyle risk factors for CRC? } \\
\hline Not at all & $6(4.1 \%)$ \\
\hline A little & $27(18.2 \%)$ \\
\hline Somewhat & $52(35.1 \%)$ \\
\hline Very much so & $63(42.6 \%)$ \\
\hline Global GAD-7 score & $4.4(3.7$ to 5.1$)$ \\
\hline Nervous/anxious & $0.8(0.6$ to 1.0$)$ \\
\hline Unable to stop/control worrying & $0.5(0.4$ to 0.7$)$ \\
\hline Worrying too much & $0.7(0.6$ to 0.9$)$ \\
\hline Trouble relaxing & $0.7(0.5$ to 0.9$)$ \\
\hline Restless & $0.4(0.3$ to 0.5$)$ \\
\hline Irritable/annoyed & 0.7 (0.6 to 0.8$)$ \\
\hline Afraid that something awful/bad might happen & $0.4(0.3$ to 0.5$)$ \\
\hline
\end{tabular}

Results are mean (95\% confidence interval) or number (percentage).

$\mathrm{CRC}=$ colorectal cancer; GAD-7 = Generalized Anxiety Disorder scale -7.

The reporting of changes in dietary changes were correlated with each other (reducing red meat/increasing fiber, fruit and vegetables $(r=0.36$ to 0.5$)$ Reporting reducing weight was correlated with decreasing red meat $(\mathrm{r}=0.55)$ and alcohol $(\mathrm{r}=0.2)$, increasing fiber $(\mathrm{r}=0.38)$, physical activity $(r=0.40)$ and fruit/vegetables $(r=0.48)$.

Increased physical activity was correlated with reducing alcohol $(\mathrm{r}=0.19)$ and weight $(\mathrm{r}=0.40)$, increasing fruit and vegetables $(r=0.55)$ and physical activity $(r=0.55)$. Decreased alcohol intake was correlated with increasing fruit and vegetable intake $(r=0.25)$ and decreased weight $(\mathrm{r}=0.20)$.

No other variables were correlated with reported changes in tobacco consumption.

\section{Influence of knowledge, risk perception and anxiety on reported behaviour change}

Global GAD-7 score and sub-scale items are shown in Table 2 . The average anxiety levels in our study population correlate with 'Mild to Moderate' anxiety [26]. The influences of perceived knowledge, risk perception and anxiety on reported behaviour change after adjustment for age, sex and socioeconomic status are shown in Table 3.

Perceived knowledge of CRC risk factors and specific worry about CRC due to family history were positively associated with reported healthy behaviour change. Higher generalized anxiety symptoms were associated with a lower number of healthy behavioural changes.

The odds of adopting at least one behaviour "Somewhat or Very much so" were 4.71 for those who worried about CRC due to family history "Often or Always", and 9.27 for those who perceived they were "Very much so" knowledgeable about CRC risk factors. The odds of adopting each behaviour "Somewhat or Very much so" were 0.66 for each SD increase in GAD-7 score.

Worry due to family history was modestly correlated with agreeing that $C R C$ was due to genetics $(r=0.33)$ and perceived knowledgeability about CRC risk $(r=0.21)$. Thoughts about the role of genetics and perceived knowledgeability not correlated.

There was no association with either NZGG-based risk, or the concordance of perceived risk and NZGGbased risk, and behaviour change. There was no association of reported adoption of healthy behaviours with CRC risk perception or whether participants believed genetics to be the primary cause of CRC.

Both GAD-7 and worry due to family history remained significantly associated with reported behavioural change when both variables were included in the model, indicating they are independent predictors of reported behavioural change.

The association between either GAD-7 or worry due to family history and healthy behaviours was not diminished when perceived knowledge of risk factors was included in a multivariate model, suggesting the mechanism by which anxiety relates to healthy behaviours is not mediated through increased knowledge of risk factors.

\section{Discussion}

Participants believed that CRC was mainly influenced by genetics, but self-reported healthy change due to concerns about CRC was still common. Participants with lower anxiety levels, higher worry due to family history of $\mathrm{CRC}$, and greater perceived knowledge of CRC risk factors were more likely to report change in lifestyle behaviours to reduce risk. Participants were more likely to report changing dietary variables and exercise, than reducing body weight, alcohol intake and tobacco smoking.

Most of our high-risk population reported adopting at least one healthy behaviour they attributed to thoughts about colorectal cancer. Patients may adopt healthy behaviours around the time of CRC screening [9], although this has been disputed $[28,29]$. The benefits of interventions to 


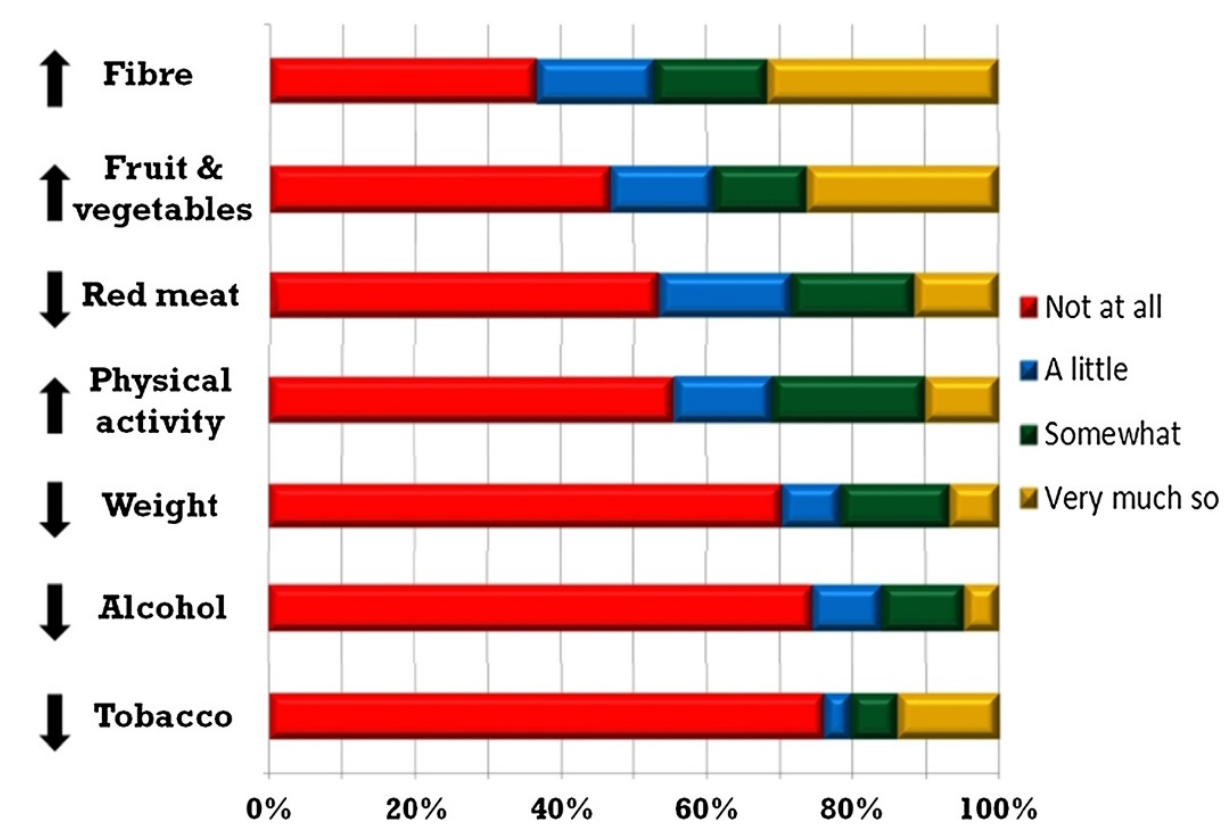

Figure 1 Self-reported change in healthy behaviours due to thoughts about colorectal cancer risk. Proportions of patients reporting each degree of adoption of specific healthy behaviours due to thoughts about colorectal cancer. Behaviours are ranked in descending order of likelihood of reported change.

produce behaviour change is small [8] compared to the normal variation of these factors amongst the general population [7]. Our findings suggest that thoughts about the risk of CRC may stimulate many patients to adopt some healthy behaviours, the exposure to the stresses associated with familial cancer and colonoscopy surveillance may represent a "teachable moment" allowing increased uptake of healthy behaviours.. Furthermore, patients may overestimate the magnitude of their lifestyle change, and further support from medical professionals may increase the likelihood that the changes made are clinically significant.

There are at least two explanations why CRC risk factors may be changed in the reported frequencies. The first is that dietary variables and physical exercise are among the most widely known risk factors in the general population [4]. Studies have found that knowledge of dietary risk factors for CRC including red meat [3,4], fruit and vegetables [4], and lack of dietary fiber [3], was good in the general population, with a smaller proportion of people knowing about smoking [3,4], and lack of physical exercise $[3,4]$ as contributing factors.

Secondly, it may be easier to change diet than to alter smoking, alcohol intake or body weight.

We investigated factors associated with self-reported change due to concerns about CRC. Perceived risk of $\mathrm{CRC}$ and true risk due to family history were not associated with healthy behaviours. Greater levels of general anxiety, specific worry about CRC due to family history and perceived knowledge about risk factors were all associated with an increased likelihood of reporting healthy behaviour adoption.

Most of our study participants had low to moderate levels of general anxiety, with few with high anxiety scores. This association between moderate levels of anxiety and adoption of healthy behaviours is consistent with previous research with breast cancer $[15,30]$. In contrast, high levels of general anxiety have been linked to maladaptive behaviour patterns [19]. However it appears that the relationship between specific worry and behavioural change is stronger and more consistent than that of general anxiety and behavioural change $[13,20,30]$. Our findings suggest that both lower general anxiety and higher specific worry were independently associated with the likelihood of reporting behavioural change. There have been limited studies on the relationship between general anxiety and self-reported behavioural change, but Bowen et al. [15] found that women with moderate levels of anxiety were more likely to report that they ate a low fat diet.

We found no association between perceived risk of $\mathrm{CRC}$ and behavioural change. Other studies have linked elevated risk perception to the uptake of cancer screening [11]. Participants were asked specifically to estimate their risk had they not undergone surveillance. Thus the lack of association between risk perception and reported behavioural change may be due to participants reappraising their risk after undergoing colonoscopy, and this risk estimate not being currently relevant to their decision-making. 
Table 3 Adjusted odds ratios for the association of knowledge, anxiety and worry with adoption of healthy behaviours

\begin{tabular}{|c|c|c|c|}
\hline & Adjusted odds ratio $\dagger$ & $95 \%$ confidence interval & $P$ value \\
\hline \multicolumn{4}{|l|}{ Perceived knowledge of $C R C$ risk factors } \\
\hline None & - & - & - \\
\hline A little & 4.16 & 0.06 to 25.04 & 0.12 \\
\hline Somewhat & 5.01 & 0.91 to 27.61 & 0.64 \\
\hline Very much so & 9.27 & 1.69 to 50.72 & 0.01 \\
\hline \multicolumn{4}{|l|}{ Are genes the main cause of CRC? } \\
\hline Not at all & - & - & - \\
\hline A little & 2.58 & 0.40 to 16.65 & 0.32 \\
\hline Somewhat & 2.71 & 0.44 to 16.65 & 0.28 \\
\hline Very much so & 3.79 & 0.61 to 23.68 & 0.15 \\
\hline Perceived risk of CRC, per SD increase & 1.30 & 0.97 to 1.75 & 0.083 \\
\hline Global GAD-7 score, per SD increase & 0.66 & 0.49 to 0.89 & 0.007 \\
\hline \multicolumn{4}{|l|}{ Worry about CRC due to family history } \\
\hline Never & - & - & - \\
\hline A little & 3.34 & 1.11 to 10.17 & 0.033 \\
\hline Sometimes & 2.96 & 1.05 to 8.33 & 0.040 \\
\hline Often/always & 4.71 & 1.55 to 14.37 & 0.006 \\
\hline
\end{tabular}

† Adjusted for age, sex, and NZDep score.

₹ Often/always combined due to low numbers reporting "always".

Odds ratios are for the number of behaviours participants rated they had changed "Somewhat" or "Very much so" compared to "Not at all" or "A little".

$\mathrm{CRC}=$ colorectal cancer; GAD-7 = Generalized Anxiety Disorder scale - 7.

Those with higher perceived risk were likely to attribute a genetic cause to CRC. This might reflect a degree of fatalism amongst those with elevated risk perception, which may have played a role in inhibiting lifestyle behavioural change [31].

Greater perceived knowledge being associated with a greater likelihood of colonoscopy screening uptake with both knowledge about the need for colonoscopy screening [32], and general knowledge about CRC [33] both being important modifying factors. We are not aware that it has been reported as influencing healthy lifestyle changes however.

\section{Limitations}

We prompted participants by enquiring whether they had adopted specific healthy behaviours, which may have led to over-reporting of healthy choices. Furthermore, a number of our questions were about subjective perception. While this provides insight into patients' thoughts, it also decreases the external validity of the findings.

This was a cross sectional study. Future studies should ideally assess the components of risk perception, anxiety and behavioural change longitudinally before, during and after participation in a surveillance program. Our study focused on patients undergoing surveillance, future studies could compare adoption of health behaviours with low risk groups.
Our study population was predominantly NZ European, potentially limiting generalization to people of other cultures.

There is no model for assessing the risk of CRC that includes measures of lifestyle risk factors that has been validated in our population or is in regular use. Calculated risk due to family history as assessed by NZGG category may not completely reflect the true risk for each individual although is the tool used in clinical practice.

Participant recall has been shown to underestimate the prevalence of CRC in the family history [34]. We validated the family history from the clinical records of each participant, but cannot be certain that we have not underestimated their true CRC risk attributable to family history.

We had a relatively good response rate, although those excluded were slightly but significantly younger than those included, raising the possibility of selection bias although it would appear unlikely to have influenced our conclusions.

\section{Implications for future practice}

Clinicians should recognize that behavioural risk factor awareness and reported attempts to change are widespread in this high-risk population and support may be indicated. Further research is required to establish risk markers for patients who are less likely to improve lifestyle factors. 


\section{Conclusion}

Our study found that participants undergoing colonoscopy surveillance for a family history of CRC perceive themselves to be knowledgeable about lifestyle risk factors for CRC and frequently report attempts to change healthy lifestyle risk factors. Lower general anxiety, higher specific worry due to family history, and perceived knowledge about CRC risk factors were independently associated with self-reported behavioural change.

\section{Abbreviations}

CRC: Colorectal cancer; NZGG: New Zealand Guidelines Group; GAD-7: Generalised Anxiety Disorder scale - 7; ANOVA: Analysis of variance; IQR: Interquartile range; NZDep: New Zealand Deprivation Index 2006; SD: Standard deviation.

\section{Competing interests}

The authors declare that they have no competing interests.

\section{Authors' contributions}

All authors were involved in the design of the study. GPT, AC, JR, JBK, HLL, HM, KMS, RS, SES and LW conducted the data collection. Statistical analysis was conducted by GPT and CC, and all authors were involved in the interpretation. The manuscript was prepared by GPT, AC, RJ, RS, CC, JDD, MS and IAM. All authors read and approved the final manuscript.

\section{Acknowledgements}

We gratefully acknowledge the funding support of the Department of Preventive and Social Medicine. The funding body had no role in the design or the conduct of the present research. We are also indebted to Nicola Casey, Department of Preventive and Social Medicine, for her secretarial assistance.

\section{Author details}

1Dunedin School of Medicine, University of Otago, PO Box 913, Dunedin, New Zealand. ${ }^{2}$ Preventive and Social Medicine, Dunedin School of Medicine, University of Otago, Dunedin, NZ. ${ }^{3}$ Gastroenterology Unit, Dunedin Public Hospital, Dunedin, NZ

Received: 20 August 2013 Accepted: 30 January 2014

Published: 7 February 2014

\section{References}

1. Chan AT, Giovannucci EL: Primary prevention of colorectal cancer. Gastroenterology 2010, 138(6):2029-2043.

2. Edwards BK, Ward E, Kohler BA, Eheman C, Zauber AG, Anderson RN, Jemal A, Schymura MJ, Lansdorp-Vogelaar I, Seeff LC, et al: Annual report to the nation on the status of cancer, 1975-2006, featuring colorectal cancer trends and impact of interventions (risk factors, screening, and treatment) to reduce future rates. Cancer 2010, 116(3):544-573.

3. Sessa A, Abbate R, Di Giuseppe G, Marinelli P, Angelillo IF: Knowledge, attitudes, and preventive practices about colorectal cancer among adults in an area of Southern Italy. BMC Cancer 2008, 8:171.

4. Shokar NK, Vernon SW, Weller SC: Cancer and colorectal cancer: knowledge, beliefs, and screening preferences of a diverse patient population. Fam Med 2005, 37(5):341-347.

5. Karthik S, Tahir N, Thakur B, Nair U: Risk factor awareness and secondary prevention of coronary artery disease: are we doing enough? Interact Cardiovasc Thorac Surg 2006, 5(3):268-271.

6. Potvin L, Richard L, Edwards AC: Knowledge of cardiovascular disease risk factors among the Canadian population: relationships with indicators of socioeconomic status. CMAJ 2000, 162(9 Suppl):S5-11.

7. Kirkegaard H, Johnsen NF, Christensen J, Frederiksen K, Overvad K, Tjonneland A: Association of adherence to lifestyle recommendations and risk of colorectal cancer: a prospective Danish cohort study. BMJ 2010, 341:C5504

8. Honda KG, D R, Neugut Al: The associations between psychological distress and cancer prevention practices. Cancer Detect Prev 2005, 29:26-36.
9. Miles A, Wardle J, McCaffery K, Williamson S, Atkin W: The effects of colorectal cancer screening on health attitudes and practices. Cancer Epidemiol Biomarkers Prev 2003, 12(7):651-655.

10. Meissner HI, Yabroff KR, Dodd KW, Leader AE, Ballard-Barbash R, Berrigan D: Are patterns of health behaviour associated with cancer screening? Am J Health Promot 2009, 23(3):168-175.

11. Kim SE, Perez-Stable EJ, Wong S, Gregorich S, Sawaya GF, Walsh JM, Kaplan CP: Association between cancer risk perception and screening behaviour among diverse women. Arch Intern Med 2008, 168(7):728-734.

12. Gimeno-Garcia A: Factors influencing colorectal cancer screening participation. Gastroenterol Res Pract 2012, 2012:483417.

13. Wardle J, Sutton S, Williamson S, Taylor T, McCaffery K, Cuzick J, Hart A, Atkin W: Psychosocial influences on older adults' interest in participating in bowel cancer screening. Prev Med 2000, 31(4):323-334.

14. Lindberg NM, Wellisch D: Anxiety and compliance among women at high risk for breast cancer. Ann Behav Med 2001, 23(4):298-303.

15. Bowen D: The relationship between perceived risk, affect, and health behaviours. Cancer Detect Prev 2004, 6(28):409-417.

16. Collins V, Halliday J, Warren R, Williamson R: Cancer worries, risk perceptions and associations with interest in DNA testing and clinic satisfaction in a familial colorectal cancer clinic. Clin Genet 2000, 58(6):460-468

17. Senore C: Comparing different strategies for colorectal cancer screening in Italy: predictors of patients' participation. Am J Gastroenterol 2010, 1(105):188-198.

18. Brain K, Norman P, Gray J, Mansel R: Anxiety and adherence to breast self-examination in women with a family history of breast cancer. Psychosom Med 1999, 61(2):181-187.

19. Kash KM, Holland JC, Halper MS, Miller DG: Psychological distress and surveillance behaviours of women with a family history of breast cancer. J Natl Cancer Inst 1992, 84(1):24-30.

20. Hay J: Does worry about breast cancer predict screening behaviours? A meta-analysis of the prospective evidence. Prev Med 2006, 42(6):401-408.

21. Parkin DM, Bray F, Ferlay J, Pisani P: Global cancer statistics, 2002. CA Cancer J Clin 2005, 55(2):74-108.

22. Stead M, Caswell S, Craigie AM, Eadie D, Anderson AS, Be WEL: Understanding the potential and challenges of adenoma treatment as a prevention opportunity: insights from the BeWEL formative study. Prev Med 2012, 54(1):97-103.

23. Dowswell G, Ryan A, Taylor A, Daley A, Freemantle N, Brookes M, Jones J, Haslop R, Grimmett C, Cheng KK, et al: Designing an intervention to help people with colorectal adenomas reduce their intake of red and processed meat and increase their levels of physical activity: a qualitative study. BMC Cancer 2012, 12:255.

24. Tarr GP, Smith RA, John RA, Crowley AP, Kok JBC, Lee H-N, Mustafa MHB, Sii $\mathrm{KM}$, Son SE, Weaver $L$, et al: Perceived risks and benefits of surveillance colonoscopy in people undergoing surveillance for family history of colorectal cancer. N Z Med J 2012, 126(1382):58-69.

25. NZDep2006 index of deprivation. http://www.health.govt.nz/system/files/ documents/publications/nzdep2006-users-manual.pdf.

26. Spitzer RL, Kroenke K, Williams JB, Lowe B: A brief measure for assessing generalized anxiety disorder: the GAD-7. Arch Intern Med 2006 166(10):1092-1097.

27. Guidance on surveillance for people at increased risk of colorectal cancer. http://nzgg.org.nz/resources/187/111206\%20Guidance\%20on\% 20Surveillance\%20forColorectal\%20Cancer\%20FINAL.pdf.

28. Brener ND, Billy JO, Grady WR: Assessment of factors affecting the validity of self-reported health-risk behaviour among adolescents: evidence from the scientific literature. J Adolesc Health 2003, 33(6):436-457.

29. Larsen IK, Grotmol T, Almendingen K, Hoff G: Impact of colorectal cancer screening on future lifestyle choices: a three-year randomized controlled trial. Clin Gastroenterol Hepatol 2007, 5(4):477-483.

30. Consedine NS, Magai C, Krivoshekova YS, Ryzewicz L, Neugut Al: Fear, anxiety, worry, and breast cancer screening behaviour: a critical review. Cancer Epidemiol Biomarkers Prev 2004, 13(4):501-510.

31. Miles A, Voorwinden S, Chapman S, Wardle J: Psychologic predictors of cancer information avoidance among older adults: the role of cancer fear and fatalism. Cancer Epidemiol Biomarkers Prev 2008, 17(8):1872-1879.

32. McLachlan SA, Clements A, Austoker J: Patients' experiences and reported barriers to colonoscopy in the screening context-a systematic review of the literature. Patient Educ Couns 2012, 86(2):137-146. 
33. McCaffery K, Wardle J, Waller J: Knowledge, attitudes, and behavioural intentions in relation to the early detection of colorectal cancer in the United Kingdom. Prev Med 2003, 36(5):525-535.

34. Mitchell RJ, Brewster D, Campbell H, Porteous ME, Wyllie AH, Bird CC,

Dunlop MG: Accuracy of reporting of family history of colorectal cancer. Gut 2004, 53(2):291-295.

doi:10.1186/1471-230X-14-22

Cite this article as: Tarr et al:: Do high risk patients alter their lifestyle to reduce risk of colorectal cancer? BMC Gastroenterology 2014 14:22.

\section{Submit your next manuscript to BioMed Central} and take full advantage of:

- Convenient online submission

- Thorough peer review

- No space constraints or color figure charges

- Immediate publication on acceptance

- Inclusion in PubMed, CAS, Scopus and Google Scholar

- Research which is freely available for redistribution 